\title{
Pregnancy benefit of acupuncture on in vitro fertilization: A systematic review and meta-analysis
}

\author{
Shiyan Yan ${ }^{1}$, Xiaoyu Liu ${ }^{2}$, Zhenqing $\mathrm{Pu}^{1}$, Yuhan Lin $^{1}$, Zhiyi Xiong ${ }^{1}$, Baoyan Liu ${ }^{3}$, Haoyan \\ Zhang ${ }^{4}$, and Cunzhi Liu ${ }^{1}$ \\ ${ }^{1}$ Beijing University of Chinese Medicine \\ ${ }^{2}$ Tianjin University of Traditional Chinese Medicine \\ ${ }^{3}$ China Academy of Chinese Medical Sciences \\ ${ }^{4}$ Chinese Academy of Medical Sciences and Peking Union Medical College
}

September 25, 2021

\begin{abstract}
Background: The evidence of acupuncture for in vitro fertilization (IVF) remains debatable. Objectives: To evaluate the efficacy of acupuncture on embryo transfer in women undergoing IVF. Search Strategy: PubMed, MEDLINE, Web of Science, EMBASE, CENTRAL, Wanfang, Chinese National Knowledge Infrastructure, VIP were searched up to 6 September 2021. Selection Criteria: Randomized controlled trials (RCTs) of acupuncture evaluating IVF's effects were included. Data Collection and Analysis: Clinical pregnancy rate (CPR) and live birth rate (LBR) were quantitatively summarized by the randomeffect model. Variations in pooled estimates were examined by subgroup analyses and I2 was measured to quantify statistical heterogeneity. Main Results: Forty-nine eligible RCTs representing 9422 women undergoing IVF for pregnant success were identified. Pooled CPR and LBR showed a significant difference between acupuncture and control groups (odds ratio [OR]=1.65, 95\% confidence interval [CI]: 1.45 to 1.88 ; OR=1.34, $95 \%$ CI: 1.08 to 1.66). Subgroup analysis showed a benefit of traditional acupuncture for women aged $<35$ years (CPR: OR=1.57, 95\% CI: 1.23 to 2.03 ; LBR: OR=1.34, 95\% CI:1.23 to 1.48), treatment with ? 3 sessions (CPR: OR=2.37, 95\% CI: 1.46 to 3.85; LBR: OR=1.99, 95\% CI:1.52 to 2.60), and lower baseline control group rates of $\mathrm{CPR}$ (CPR: $\mathrm{OR}=2.14,95 \% \mathrm{CI}: 1.22$ to 2.13 ; LBR: $\mathrm{OR}=2.23,95 \% \mathrm{CI}: 1.07$ to 4.62 ). Heterogeneity across studies were found (CPR: $\mathrm{I} 2=47.4, \mathrm{p}<0.001 ; \mathrm{LBR}$ : $\mathrm{I} 2=62.9, \mathrm{p}<0.001)$. Conclusions: Although benefits of acupuncture in CPR and LBR among IVF women existed, no robust associations were found and which might be limited by the heterogeneity of current evidence
\end{abstract}

\section{Introduction}

Infertility is an important public health issue and approximately from $9 \%$ to $18 \%$ of couples worldwide suffering from infertility (1-3). For achieving pregnancy, in vitro fertilization (IVF) has become a widespread treatment option. Despite many recent technological advances, the average success rate of IVF was only $36 \%$ reported by the Centers for Disease Control and Prevention of USA at 2016 (4). Acupuncture has been used as an attractive and most frequently adjuvant therapy based on its low side-effects and convenience (5). A study conducted in USA showed that $22 \%$ couples had used acupuncture (6). However, evidence from acupuncture in improving IVF birth outcomes still remains a matter of debate.

Since the first randomized clinical trial (RCT) was published in 1999 (7), nearly 50 RCTs have emerged to explore acupuncture's impact on IVF outcomes. However, the conclusions in inconclusive. Even if many systematic reviews and meta-analyses still revealed both positive effects (8-15) and negative benefits of 
acupuncture assisting IVF success (16-24). Different searching databases and literature selection criteria may partly contribute to the unconfirmed results. For example, some analyses $(11,14,16,17)$ just included English language literature. Several analyses did not cover all the acupuncture types or control (12, 14, $22)$. In addition, most of meta-analyses considered clinical pregnancy rate (CPR) as a primary outcome and neglected the live birth rate (LBR). LBR was an accepted primary outcome in IVF trials $(25,26)$ and its related results need to be further updated. The conflicted results might be not due to the ineffectiveness of acupuncture but rather to the methodological flaws confound acupuncture IVF RCTs. Therefore, taken together with the continuously added new evidence, this study aimed to evaluate the impact of acupuncture on IVF outcomes for both CPR and LBR based on RCTs by conducting a comprehensive literature covered English and Chinese involving all common acupuncture methods.

\section{Methods}

\section{Literature search}

PubMed, MEDLINE, Web of Science, EMBASE and Cochrane Register of Controlled Clinical Trials (CENTRAL) were searched to obtain articles addressing the effect of acupuncture on the outcomes of IVF-ET published up to 6 September 2021. Medical Subject Headings (MeSH) terms and free text terms were combined to screen for potentially relevant studies in PubMed, the detailed search algorithm will be slightly modified and used in several other databases (Appendix S1). In addition, some Chinese databases such as the Wanfang Databases, Chinese National Knowledge Infrastructure (CNKI), the VIP databases and SinoMed were used to collect related studies in China.

Other potentially relevant papers were also identified by cross-referencing. The present study complied with the preferred reporting items for systematic reviews and meta-analyses (PRISMA) guidelines 6 (Table S1 ) .

Inclusion criteria

The inclusion criteria were as follows: (1) RCTs that evaluated the IVF's effects for women; (2) articles were published in English or Chinese; (3) pregnant data could be directly extracted or calculated from the original article; (4) any type of acupuncture was accepted, including traditional acupuncture (TA), electrical acupuncture (EA), warm acupuncture (WA), auricular acupuncture (AA), laser acupuncture (LA), transcutaneous electrical acupoint stimulation (TEAS) and others. However, if there were one or above treatments together with acupuncture in treatment group, the other treatments between intervention and control group should be the same. No treatment, sham or placebo acupuncture, active control including Western medicines, dietary supplements or lifestyle interventions were all allowed.

If the study was reported in duplication, the version firstly published or provided more detailed information was included. Studies with male factor infertility, review articles, animal studies, abstracts and case reports were excluded.

Data extraction

All of the potentially relevant literature were reviewed independently by two investigators and consensus was reached on all of the items. Any disagreements were resolved by discussion or with involvement of a third review author. CPR (presence of at least one gestational sac or fetal heartbeat, confirmed by transvaginal ultrasound) and LBR (a baby born alive after 24 weeks gestation) were the primary outcomes, and the secondary outcomes were ongoing pregnancy rate (OPR, pregnancy beyond 12 weeks of gestation, as confirmed by fetal heart activity on ultrasound), miscarriage rate (MR) and adverse events related to acupuncture. The following information was also needed: first author and published year, sample size, age of the study participants, acupuncture sessions or timing.

Assessment for risk of bias 
The potential methodological biases were assessed using the Risk of Bias (RoB) tool that evaluated seven parameters (27). Each item of every trial was categorized as "high risk $(\mathrm{H})$ ", "unclear (U)", or "low risk (L)". If two of the domains were rated as high, the study was considered to be at high risk of bias.

Statistical analysis

Meta-analyses were carried out using STATA Meta-Analysis (V2.0, Biostat, Englewood, NJ, USA). The RoB graph was drawn by Review Manager 5.3 (Cochrane Collaboration Software, RevMan). The measure of treatment effect was calculated by the pooled odds ratio (OR) with $95 \%$ confidence intervals (CI) with random effects models. Intention-to-treat (ITT) analysis would be used when available. The number of trials in meta-analysis might not always be 49 , because the included studies had multiple treatment or control types and the meta-analysis was undertaken based on each comparison.

Subgroup and meta-regression analyses were predefined with respect to age of participants $(<35$ or [?]35 years (28) ), different acupuncture types, type of control (no treatment or sham/placebo control), acupuncture timing (two types were categorized: type A, the acupuncture was performed around the time of ET (embryo transfer), which referred to the acupuncture treatment performed before and after ET (29); type B, before the ET); geographical origin of the study (Asian or not Asian), whether or not RIF (repeated IVF failure), acupuncture sessions (1,2 or more than 3 sessions). Otherwise, the clinical characteristic previously was found to benefit trials with lower baseline pregnancy rates $(20,30)$, therefore, the stratified analysis was conducted by the pregnancy rate of no treatment group. Sensitivity analyses were also conducted to explore the robustness of the pooled effects of included studies: (1) restricting CPR results to the studies which simultaneously reporting LBR; (2) limiting the studies with low risk of bias; (3) deleting the studies whose primary outcome was not to evaluate the effects of acupuncture on pregnancy rates from IVF.

Heterogeneity was assessed using the $I^{2}$ test. Publication bias were shown by a funnel plot and assessed by the Egger weighted regression methods ( $p<0.05$ was considered statistically significant).

\section{Results}

\section{Search results}

The selection process was presented in Figure S1. The initial search identified 1742 articles. After duplicate articles removed, 1018 were screened. Based on the title and abstract, 101 articles were further detailed evaluated. Then 52 articles were excluded and 49 RCTs (9422 participants) with 29 published in English $(7,30-57)$ and 20 in Chinese (58-77) were included in the final review (Table S2 ).

Risk of bias of included studies

As shown in Table S3 andFigure S2, there were no specific descriptions about random sequence generation in nine studies $(31,48,50,60,65,72,74,77)$ and they were determined to be "unclear". There were 5 studies with both a sham group and a no treatment control $(35,40,64,74,77)$ which were defined as unclear risk for blinding of participants. However, studies with no treatment control were scored as high risk of bias. In all included studies, there was no attrition bias from reviewing methods and results. Since results could be directly obtained, reporting bias was assessed as 'low risk' for all included studies. In addition, other sources of bias such as improper funding were not described and were at unclear risk of this bias.

\section{Study characteristics}

Study characteristics were showed in Table 1 . Eighteen studies used TA alone (30, 32, 33, 43-46, 51-53, $58,61,62,64,67,74,75,77), 6$ studies involved the administration of WA alone $(59,60,66,70,71,73)$, 6 for EA alone $(7,39,49,50,63,76), 5$ for TEAS alone $(33,34,37,38,41)$ and the remaining fourteen studies had two treatment groups or combined with two interventions in a treatment group. Only sham or placebo acupuncture was administered in 11 studies $(30,33,38,41,43,44,46,51,53,54,70)$, and 24 studies used only a no treatment control $(7,25,31,32,34,39,45,47,49,50,52,58-63,65,66,71-73,75$, 
$76)$. Ten trials included three arms $(31,40,41,48,52,54,63,64,74,77)$ and 4 trials were four-arms $(34$, $35,37,67)$. Among them, both sham control and no treatment control were used in 5 trials $(35,40,64,74$, 77). Seven studies included women who were RIF patients $(33,38,40,61,65,66,71)$. Acupuncture was provided before the ET among 8 studies and 20 studies around the ET. The acupuncture sessions differed across studies. Regarding TA treatment, 2 studies performed one acupuncture session, 17 studies performed two acupuncture sessions and 9 studies performed three or above sessions. CPR was available from all the 49 trials. 13 reported LBR $(30,32-35,38,41,43-46,50,60,74)$ and 10 studies provided OPR data $(31,32$, $39,43,44,46,51-53,56)$. 27 studies were performed in China (33, 34, 37, 38, 41, 44, 46, 49, 55-74, 76, 77), 9 in Europe $(7,35,43,51,52,54-56,75), 4$ in USA $(42,47,48,50)$, and the remaining were in other country. The main objective in 4 studies was to assess pain relief $(7,54-56)$, and the remaining studies were all for improvement of IVF outcomes. Total samples sizes for each study ranged from 44 to 848 women.

Acupuncture effects on IVF outcomes

As shown in Table 1, pooled CPR and LBR data showed a significant difference between acupuncture and control groups $(60$ trials, $\mathrm{OR}=1.65,95 \% \mathrm{CI}$ : 1.45 to $1.88 ; 20$ trials, $\mathrm{OR}=1.34,95 \% \mathrm{CI}$ : 1.08 to 1.66 , respectively). However, acupuncture had no significant improvement for OPR (11 trials, OR=1.33, 95\%CI=0.93 to 1.89) (Table S4 ). Table S5 showed the subgroup analysis for CPR of TA by control type.

As for the effects of different acupuncture methods and shown inTable $\mathbf{1}$, only TEAS was found to be a positive effect for both CPR ( 10 trials, $\mathrm{OR}=1.37,95 \% \mathrm{CI}=1.21$ to 1.56$)$ and $\mathrm{LBR}$ ( 7 trials, $\mathrm{OR}=1.74$, $95 \% \mathrm{CI}=1.23$ to 2.46$)$. In addition, 28 studies used $\mathrm{TA}(\mathrm{OR}=1.24,95 \% \mathrm{CI}=1.15$ to 1.33$), 6$ studies used WA $(\mathrm{OR}=1.75,95 \% \mathrm{CI}=1.33$ to 2.32$), 7$ studies used $\mathrm{EA}(\mathrm{OR}=1.45,95 \% \mathrm{CI}=1.13$ to 1.85$)$ and 2 studies used LA $(\mathrm{OR}=1.22,95 \% \mathrm{CI}=1.06$ to 1.41$)$ showed a positive improvement for $\mathrm{CPR}$ results. 33 trials reported $\mathrm{MR}$ and statistically significant difference was found between TA and no treatment group (OR=1.44, 95\% $\mathrm{CI}=1.00$ to 2.07) (Figure S3 ). Table S6 showed the risk of adverse events were higher in acupuncture $(6.59 \%$ vs $1.09 \%, \mathrm{p}<0.001)$ than no treatment group, but no significant difference compared with sham or placebo acupuncture $(47.61 \%$ vs $44.26 \%, \mathrm{p}=0.241)$.

Subgroup analyses

Since there was statistical heterogeneity for IVF outcomes across studies (CPR: $I^{2}=47.4, \mathrm{p}<0.001 ;$ LBR: $I^{2}$ $=62.9, \mathrm{p}<0.001$ ), subgroup analyses and random-effects meta-regression were utilized. In subgroups classified by treatment type, studies used TA was found to be statistical heterogeneity (CPR: $I^{2}=61.5, \mathrm{p}<0.001$; LBR: $I^{2}=68.3, \mathrm{p}=0.001$ ) (Table 1 ). Therefore, the meta-regression analyses were restricted to TA methods. With regard to TA and TEAS on CPR, control type (no acupuncture, sham or placebo) was found to be the potential source of heterogeneity ( $\mathrm{p}=0.043$, respectively) (Table 2 ). When compared with no treatment, TA did improve $\mathrm{CPR}$ ( 18 trials, $\mathrm{OR}=1.85,95 \% \mathrm{CI}=1.51$ to 2.28$)$. However, no significant difference was found between TA and sham control (penetration: 4 trials, $\mathrm{OR}=1.89,95 \% \mathrm{CI}=0.93$ to 3.82 ; non-penetration: 6 trials, $\mathrm{OR}=1.04,95 \% \mathrm{CI}=0.76$ to 1.43$)$. Similar findings were observed for LBR results, TA group was significantly higher than that from no treatment ( 10 trials, $\mathrm{OR}=1.36,95 \% \mathrm{CI}=1.07$ to 1.73 ), but not from sham control group ( 9 trials, $\mathrm{OR}=1.42,95 \% \mathrm{CI}=0.95$ to 2.11$)$. While according to acupuncture sessions, TA performed for 2 or more than 3 times showed significant effect on CPR ( 17 trials, OR=1.51, $95 \% \mathrm{CI}=1.18$ to $1.94 ; 9$ trials, $\mathrm{OR}=1.99,95 \% \mathrm{CI}=1.52$ to 2.60 ; respectively), while performed for 1 session found no significance ( 2 trials, $\mathrm{OR}=1.47,95 \% \mathrm{CI}=0.34-6.39$ ). It was also an increasing improvement for TEAS if three or more treatments were administered ( 5 trials, $\mathrm{OR}=2.34,95 \% \mathrm{CI}=1.58$ to 3.48 ) (Tables 2-3 ). The additional results of the effects of different acupuncture methods by baseline CPR, OPR or LBR of no treatment group were shown inFigure S4. Studies with lower control group rates of CPR showed larger effects of TA $(\mathrm{OR}=2.14,95 \% \mathrm{CI}=1.63$ to 2.81$)$ than that with higher control group rates of $\mathrm{CPR}(\mathrm{OR}=1.61$, $95 \% \mathrm{CI}=1.22$ to 2.13$)$.

Sensitivity analyses

Table S7 showed the results of sensitivity analysis. When the analysis was restricted to "low risk of bias" studies, for TA treatment, the overall pooled CPR results returned to no difference (before: OR=1.24, 
95\% CI: 1.15 to 1.33; after: $\mathrm{OR}=1.13,95 \% \mathrm{CI}: 0.98$ to 1.31 ), but the LBR results were unchanged (before: $\mathrm{OR}=1.18,95 \% \mathrm{CI}$ : 0.86 to 1.60 ; after: $\mathrm{OR}=0.95,95 \%$ CI: 0.80 to 1.13 ). The pooled CPR (before: $\mathrm{OR}=1.37$, 95\% CI: 1.21 to 1.56; after: $\mathrm{OR}=1.58,95 \%$ CI: 1.13 to 2.19 ) and LBR (before: $\mathrm{OR}=1.74,95 \%$ CI: 1.23 to 2.46 ; after: $\mathrm{OR}=1.62,95 \% \mathrm{CI}: 1.26$ to 2.07 ) results remained stable for TEAS before and after sensitivity analysis. When restricting to studies which reported both CPR and LBR, no significant difference was found for the pooled CPR results ( $\mathrm{OR}=1.14,95 \% \mathrm{CI}$ : 0.97 to 1.32 ).

\section{Publication bias}

Evident publication bias was observed on both CPR and LBR results as evaluated by Egger's weighted regression analysis $(\mathrm{p}<0.001$ and $\mathrm{p}=0.009)$ and funnel plot (Figure S5 ).

\section{Discussion}

\section{Main findings}

The summarized estimation of results based on $49 \mathrm{RCTs}$ showed that acupuncture might be a beneficial treatment for infertile women undergoing IVF. TEAS showed a significant benefit on both CPR and LBR improvement, while TA, WA, EA and LA were found significant effectiveness in CPR, but not significant for LBR. Subgroup analyses showed that the lower baseline estimate of the pregnancy rate had benefits on CPR and LBR.

\section{Strengths and Limitations}

The strengths of our study were including the large number of trials and their relatively sample sizes; comprehensive literature covered English and Chinese; involving all common acupuncture types (TA, EA, TEAS, WA and LA); more detailed subgroup analyses and meta regression to explore the heterogeneous; adherence to the intention to treat principle; as well as robustness of the results to sensitivity analyses. Compared with the previous and latest reviews published by Coyle ME et al ( 8 trials, English-language literature searched up to 3 April 2019) (22) and Xie ZY et al. (27 trials, literature searched up to December 2018) (15), we included the largest sample size and most comprehensive trials. Twenty trials were added in our reviews; eleven had positive results $(6,35,39,40,46,47,49,51,52,57,77)$, and nine had negative results $(30,42-45,53-56)$. In addition, we defined both CPR and LBR as primary outcomes, which were stricter than CPR alone used in almost all previous reviews. Compared to CPR, LBR is more likely to reflect the endpoint of IVF therapy and the most concerned outcome for patients.

A limitation of this review was the significant heterogeneous among trials. The acupuncture prescription and control type might be the important contributions to the heterogeneous. Although most acupuncture prescriptions were generally standardized based on the original protocol of Paulus et al (57), nearly every study would make appropriate adjustments to some extent (20). In addition, the type of control especially for the sham or placebo should be noted. Acupuncture point location and needle insertion or not are the main factors causing variance when we establish a sham or placebo acupuncture. In traditional Chinese medicine theory, acupoint is not a point and has its own domain. Even though a sham needle is applied away from the acupoint, it might have the similar treatment effect as the actual acupuncture. Even though noninvasive placebo acupuncture such as Streitberger acupuncture may also have certain effects because it stimulates the skin. Those have been illustrated by our results which suggested CPR and LBR in TA group were significant compared with no treatment, but no difference compared with sham or placebo acupuncture. Hence, placebo acupuncture might not always be an inert control and it is greatly difficult to establish an ideal placebo acupuncture.

\section{Interpretation}

Our review suggested that the pooled CPR and LBR from all the acupuncture group were significantly higher than that from all the control group $(\mathrm{p}<0.001)$. It was consistent with the studies reported by 
Manheimerand E et al (2008) (8) and Zheng CH et al (2012) (9). However, when restriction to only low risk of bias studies, there was no benefit of TA method on CPR and LBR. It suggested that we should caution against the benefits of TA being overstated. Of note, when only low risk of bias studies were included, a change to a non-significant effect did occur for TEAS. Because of the later published time, TEAS intervention did not involve in the existed reviews and this was the first time to report the pooled effect of TEAS. The results of TEAS is of great clinically significance for IVF, based on its advantages of noninvasiveness and painlessness, TEAS may have better application potential and be more acceptable. The safety of TEAS may be better than TA, because the MR was not significant between TEAS and no treatment. Meanwhile, unlike TA method, sham TEAS is easy to be implemented by "pseudo-percutaneous patch" method.

In our meta-analysis, TA did not increase the CPR compared with sham acupuncture, whether or not the sham needles were penetrated. This was consistent with a growing concern that sham acupuncture was not an inert control and may result in the difference between intervention and sham group not being significant. Regardless of either true or sham acupuncture was used, they could both produce specific effects and non-specific effects. The non-specific effects between treatment and sham acupuncture may be reduced to the greatest extent through blind and random methods. Therefore, in order to improve the reliability, an appropriate control group should be selected by the specific study objective like examining the pragmatic or comparative effectiveness (22).

In our study, differences were seen when different acupuncture sessions were analyzed. A dosage of [?]3 times for TA and TEAS were both associated with statistically significant improvement on CPR and LBR. Many trials tended to use two or a maximum of three sessions of acupuncture, such as "Paulus acupuncture protocol" performed just twice with one before and another after ET (57). Insufficient acupuncture dosage in IVF has been a common opinion (78). Some investigators suggested that a clinically valid dosage of acupuncture should be more than six acupuncture sessions and at least choose four to ten (or more) acupuncture points in each session (79). Further study should explore the specific effective dose of acupuncture treatment.

Our review analyzed all common acupuncture types in IVF treatment based on the most comprehensive trials. The results of TEAS supply a superior and safe choice for patients and physicians, especially compared with manual acupuncture, its advantages of noninvasiveness and easier administration partly reduce the involvement of many confounding factors cause by acupuncturists. Therefore, it may be a promising IVF adjunctive treatment and has broader application after being validated by strict RCTs. We also found that less than 35 years, treatment with [?]3 sessions, acupuncture before the ET, no RIF history and lower baseline pregnancy rates were associated with increases in CPR and LBR. These results were greatly helpful for the design of further acupuncture treatment trials in IVF. In addition, LBR should be considered as a primary outcome of the further IVF meta-analysis study. It was undeniable that clinical pregnancy was a major breakthrough in medicine, but for infertile couples, "take-home" babies were the most important long-term and ultimate outcome of interest $(25,26)$. Moreover, the efficacy was significant just for CPR rather than LBR, which may indicate a higher risk of miscarriage. This not only increased the patients' economic burden, but also further resulted in psychological consequences.

\section{Conclusion}

Our analysis indicated that acupuncture especially for TEAS improved CPR and LBR among IVF women. However, we should be prudent to interpret its clinical meanings because of the associations seemingly suggested not a substantially significant benefit. Given the numerous heterogeneity among acupuncture IVF RCTs, more robust evidences from optimal trials are needed to clarify the value of acupuncture to IVF outcomes. 


\section{Acknowledgements}

None.

\section{Disclosure of Interests}

The authors declare that the research was conducted in the absence of any commercial or financial relationships that could be construed as a potential conflict of interest.

\section{Contribution to Authorship}

HRZ, CZL, SYY, and BYL conceived the study and wrote the protocol. HRZ performed the literature search and drafted the manuscript. XYL, ZQP, ZYX, and YHL participated in the data extraction and quality control. HRZ and SYY performed the statistical analysis. All authors revised the manuscript and approved the final version. SYY is the guarantor for this work and accepts full responsibility for the conduct of the study, had access to the data, and controlled the decision to publish. The corresponding author (HRZ) attests that all listed authors meet authorship criteria and that no others meeting the criteria have been omitted.

\section{Details of Ethics Approval}

This was a systematic review of previously published trials and as such did not require formal ethics approval.

\section{Funding}

This work was supported by the National Key R\&D Program of China (2019YFC1712100) held by CZL. The funder had no role in the design and conduct of the study; collection, management, analysis, and interpretation of the data; preparation, review, or approval of the manuscript; and decision to submit the manuscript for publication.

\section{Data Availability Statement}

The datasets [GENERATED/ANALYZED] for this study can be requested from the first author atyanshiyan0927@sina.com.

\section{References}

1. Lindsay TJ, Vitrikas KR. Evaluation and treatment of infertility.Am Fam Physician. (2015) 91:308-14.

2. Hanson B, Johnstone E, Dorais J, Silver B, Peterson CM, Hotaling J. Female infertility, infertilityassociated diagnoses, and comorbidities: a review. J Assist Reprod Genet. (2017) 34:167-77.

3. Xie ZY, Peng ZH, Yao B, Chen L, Mu YY, Cheng J, et al. The effects of acupuncture on pregnancy outcomes of in vitro fertilization: a systematic review and meta-analysis. BMC Complement Altern Med . (2019) 19:131. 
4. National Center for Chronic Disease Prevention and Health Promotion, Centers for Disease Control and Prevention, with American Society for Reproductive Medicine, Society for Assisted Reproductive Technology. 2016 Assisted Reproductvie Technology National Summary Report. Atlanta, GA: U.S. Department of Health and Human Services; 2018. Online document at: www.cdc.gov/art/pdf/2016report/ART-2016-National-Summary-Report.pdf Accessed November 15, 2019.

5. Nandi A, Shah A, Gudi A, Homburg R. Acupuncture in IVF: A review of current literature. J Obstet Gynaecol. (2014) 7: 555-61.

6. Smith JF, Eisenberg ML, Millstein SG, Nachtigall RD, Shindel AW, Wing H, et al. The use of complementary and alternative fertility treatment in couples seeking fertility care: data from a prospective cohort in the United States. Fertil Steril. (2010) 93:2169-74.

7. Stener-Victorin E, Waldenström U, Nilsson L, Wikland M, Janson PO. A prospective randomized study of electro-acupuncture versus alfentanil as anaesthesia during oocyte aspiration in in-vitro fertilization. Hum Reprod. (1999) 14:2480-4.

8. Manheimer E, Zhang G, Udoff L, Haramati A, Langenberg P, Berman BM, et al. Effects of acupuncture on rates of pregnancy and live birth among women undergoing in vitro fertilisation: systematic review and meta-analysis. BMJ. (2008) 336:545-9.

9. Zheng CH, Huang GY, Zhang MM, Wang W. Effects of acupuncture on pregnancy rates in women undergoing in vitro fertilization: a systematic review and meta-analysis. Fertil Steril. (2012) 97:599-611.

10. Zheng CH, Zhang MM, Huang GY, Wang W. The role of acupuncture in assisted reproductive technology. Evid Based Complement Alternat Med. (2012) 2012:543924.

11. Qian Y, Xia XR, Ochin H, Huang C, Gao C, Gao L, et al. Therapeutic effect of acupuncture on the outcomes of in vitro fertilization: a systematic review and meta-analysis. Arch Gynecol Obstet.(2017) 295:543-58.

12. Jo J, Lee YJ. Effectiveness of acupuncture in women with polycystic ovarian syndrome undergoing in vitro fertilisation or intracytoplasmic sperm injection: a systematic review and meta-analysis. Acupunct Med . (2017) 35:162-70.

13. Li M, Liu Y, Wang H, Zheng S, Deng Y, Li Y. The Effects of acupuncture on pregnancy outcomes of recurrent implantation failure: a systematic review and meta-analysis. Evid Based Complement Alternat Med.(2021)2021:6661235.

14. Smith CA, Armour M, Shewamene Z, Tan HY, Norman RJ, Johnson NP. Acupuncture performed around the time of embryo transfer: a systematic review and meta-analysis. Reprod Biomed Online. (2019) 38:364-79.

15. Xie ZY, Peng ZH, Yao B, Chen L, Xia YB. The effects of acupuncture on pregnancy outcomes of in vitro fertilization: a systematic review and meta-analysis. BMC Complem Altern M. (2019) 9:131.

16. El-Toukhy T, Sunkara SK, Khairy M, Dyer R, Khalaf Y, Coomarasamy A. A systematic review and meta-analysis of acupuncture in in vitro fertilisation. BJOG. (2008)115:1203-13.

17. Schwarze JE, Ceroni JP, Ortega-Hrepich C, Villa S, Crosby J, Pommer R. Does acupuncture the day of embryo transfer affect the clinical pregnancy rate? Systematic review and meta-analysis. JBRA Assist Reprod. (2018) 22:363-8.

18. Sunkara SK, Coomarasamy A, Khalaf Y, El-Toukhy T. Acupuncture and in vitro fertilization: updated meta-analysis. Human Reproduction.(2009) 24:2047-8.

19. Qu F, Zhou J, Ren RX. Effects of acupuncture on the outcomes of in vitro fertilization: a systematic review and meta-analysis. J Altern Complement Med. (2012) 18:429-39.

20. Manheimer E, van der Windt D, Cheng K, Stafford K, Liu J, Tierney J, et al. The effects of acupuncture on rates of clinical pregnancy among women undergoing in vitro fertilization: a systematic review and meta-analysis. Hum Reprod Update. (2013) 19:696-713.

21. Shen C, Wu M, Shu D, Zhao X, Gao Y. The role of acupuncture in in vitro fertilization: a systematic review and meta-analysis. Gynecol Obstet Invest. (2015) 79:1-12.

22. Coyle ME, Stupans I, Abdel-Nour K, Ali H, Kotlyarsky M, Lie P, et al. Acupuncture versus placebo acupuncture for in vitro fertilisation: a systematic review and meta-analysis. Acupunct Med. (2020) 9:20-9. 
23. Cheong YC, Dix S, Hung Yu Ng E, Ledger WL, Farquhar C. Acupuncture and assisted reproductive technology. Cochrane Database Syst Rev.(2013) 7:CD006920.

24. Cheong Y, Nardo LG, Rutherford T, Ledger W. Acupuncture and herbal medicine in in vitro fertilisation: a review of the evidence for clinical practice. Hum Fertil (Camb). (2010) 13:3-12.

25. Evers JL. Female subfertility. Lancet. (2002) 360:151-9.

26. Gao R, Guo B, Bai J, Wu Y, Wu K. Acupuncture and clomiphene citrate for anovulatory infertility: a systematic review and meta-analysis. Acupunct Med. (2020) 38:25-36.

27. Higgins JP, Altman DG, Gøtzsche PC, Jüni P, Moher D, Oxman AD, et al. The Cochrane Collaboration's tool for assessing risk of bias in randomised trials. BMJ. (2011) 343: d5928.

28. Humaidan P, Marca AL, Alviggi C, Esteves SC, Haahr T. Future perspectives of POSEIDON stratification for clinical practice and research. Front Endocrinol. (2019) 10:439.

29. Han JS. Acupuncture and endorphins. Neurosci Lett. (2004) 361:258-61.

30. Smith CA, de Lacey S, Chapman M, Ratcliffe J, J Norman R, P Johnson N, et al. Effect of acupuncture vs sham acupuncture on live births among women undergoing In vitro fertilization: a randomized clinical trial.JAMA. (2018) 319:1990-8.

31. Dehghani AS, Homayouni K, Kanannejad Z, Kanannejad Z. The effect of acupuncture on the day of embryo transfer on the in vitro fertilization outcomes: An RCT. Int J Reprod Biomed (Yazd).(2020) 18:209-14.

32. Guven PG, Cayir Y, Borekci B. Effectiveness of acupuncture on pregnancy success rates for women undergoing in vitro fertilization: A randomized controlled trial. Taiwan J Obstet Gynecol . (2020) 59:282-6.

33. Shuai Z, Li X, Tang X, Lian F, Sun Z. Transcutaneous electrical acupuncture point stimulation improves pregnancy outcomes in patients with recurrent implantation failure undergoing in vitro fertilisation and embryo transfer: a prospective, randomised trial. Acupunct Med . (2019) 37:33-9.

34. Qu F, Wang FF, Wu Y, Zhou J, Robinson N, Hardiman PJ, et al. Transcutaneous Electrical Acupoint Stimulation Improves the Outcomes of In Vitro Fertilization: A Prospective, Randomized and Controlled Study. Explore (NY) . (2017) 13:306-12.

35. Morin SJ, Frattarelli JL, Franasiak JM, Juneau CR, Scott RT. Laser Acupuncture before and after Embryo Transfer Improves inVitro Fertilization Outcomes: A Four-Armed Randomized Controlled Trial.Med Acupunct. (2017) 29:56-65.

36. Cochrane S, Smith CA, Possamai-Inesedy A, Bensoussan A. Prior to Conception: The Role of an Acupuncture Protocol in Improving Women's Reproductive Functioning Assessed by a Pilot Pragmatic Randomised Controlled Trial. Evid Based Complement Alternat Med.(2016)2016:3587569.

37. Zheng Y, Feng X, Mi H, Yao Y, Zhao Y, Li J, et al. Effects of transcutaneous electrical acupoint stimulation on ovarian reserve of patients with diminished ovarian reserve in in vitro fertilization and embryo transfer cycles. J Obstet Gynaecol Res . (2015) 41:1905-11.

38. Shuai Z, Lian F, Li P, Yang W. Effect of transcutaneous electrical acupuncture point stimulation on endometrial receptivity in women undergoing frozen-thawed embryo transfer: a single-blind prospective randomised controlled trial. Acupunct Med. (2015) 33:9-15.

39. Rashidi BH, Tehrani ES, Hamedani NA, Pirzadeh L. Effects of acupuncture on the outcome of in vitro fertilisation and intracytoplasmic sperm injection in women with polycystic ovarian syndrome. Acupunct Med. (2013) 31:151-6.

40. Villahermosa DI, Santos LG, Nogueira MB, Vilarino FL, Barbosa CP. Influence of acupuncture on the outcomes of in vitro fertilisation when embryo implantation has failed: a prospective randomised controlled clinical trial. Acupunct Med . (2013) 31:157-61.

41. Zhang R, Feng XJ, Guan Q, Cui W, Zheng Y, Sun W, et al. Increase of success rate for women undergoing embryo transfer by transcutaneous electrical acupoint stimulation: a prospective randomized placebo-controlled study.Fertil Steril. (2011) 96:912-6.

42. Moy I, Milad MP, Barnes R, Confino E, Kazer RR, Zhang X. Randomized controlled trial: effects of acupuncture on pregnancy rates in women undergoing in vitro fertilization. Fertil Steril. (2011) 95:583-7. 
43. Andersen D, Løssl K, Nyboe AA, Fürbringer J, Bach H, Simonsen J, et al. Acupuncture on the day of embryo transfer: a randomized controlled trial of 635 patients. Reprod Biomed Online. (2010) 21:366-72.

44. So EW, Ng EH, Wong YY, Yeung WS, Ho PC. Acupuncture for frozen-thawed embryo transfer cycles: a double-blind randomized controlled trial.Reprod Biomed Online. (2010) 20:814-21.

45. Madaschi C, Braga DPAF, Figueira RDCS, Iaconelli AJ, Borges EJ. Effect of acupuncture on assisted reproduction treatment outcomes. Acupunct Med . (2010) 28:180-4.

46. So EW, Ng EH, Wong YY, Lau EY, Yeung WS, Ho PC. A randomized double-blind comparison of real and placebo acupuncture in IVF treatment. Hum Reprod. (2009) 24:341-8.

47. Domar AD, Meshay I, Kelliher J, Alper M, Powers RD. The impact of acupuncture on in vitro fertilization outcome. Fertil Steril . (2009) 91:723-6.

48. Kong S, Hughes A. Acupuncture as an adjunct to in vitro fertilization: a randomized trial. Med Acupunct . (2009) 21:179-82.

49. Ho M, Huang LC, Chang YY, Chen HY, Chang WC, Yang TC, et al. Electroacupuncture reduces uterine artery blood flow impedance in infertile women. Taiwan J Obstet Gynecol . (2009) 48:148-51.

50. Magarelli PC, Cridennda DK, Cohen M. Changes in serum cortisol and prolactin associated with acupuncture during controlled ovarian hyperstimulation in women undergoing in vitro fertilizationembryo transfer treatment. Fertil Steril. (2009) 92:1870-9.

51. Dieterle S, Ying G, Hatzmann W, Neuer A. Effect of acupuncture on the outcome of in vitro fertilization and intracytoplasmic sperm injection: a randomized, prospective, controlled clinical study.Fertil Steril. (2006) 85:1347-51.

52. Westergaard LG, Mao Q, Krogslund M, Sandrini S, Lenz S, Grinsted J. Acupuncture on the day of embryo transfer significantly improves the reproductive outcome in infertile women: a prospective, randomized trial. Fertil Steril. (2006) 85:1341-6.

53. Smith C, Coyle M, Norman RJ. Influence of acupuncture stimulation on pregnancy rates for women undergoing embryo transfer. Fertil Steril. (2006) 85:1352-8.

54. Sator-Katzenschlager SM, Wölfler MM, Kozek-Langenecker SA, Sator K, Sator PG, Li B, et al. Auricular electro-acupuncture as an additional perioperative analgesic method during oocyte aspiration in IVF treatment. Hum Reprod. (2006) 21:2114-20.

55. Humaidan P, Stener-Victorin E. Pain relief during oocyte retrieval with a new short duration electroacupuncture technique-an alternative to conventional analgesic methods. Hum Reprod.(2004) 19:136772.

56. Stener-Victorin E, Waldenstrom U, Wikland M, Nilsson L, Hagglund L, Lundeberg T. Electroacupuncture as a peroperative analgesic method and its effects on implantation rate and neuropeptide Y concentrations in follicular fluid. Hum Reprod. (2003) 18:1454-60.

57. Paulus WE, Zhang M, Strehler E, El-Danasouri I, Sterzik K. Influence of acupuncture on the pregnancy rate in patients who undergo assisted reproduction therapy. Fertil Steril. (2002) 77:721-4.

58. Wu JM, Zhuo YY, Tang M, Hu S, Ye YY, Ning Y. Effect of acupuncture on the outcome of in vitro fertilization among patients with polycystic ovarian syndrome. Shanghai J Acu-mo . (2020):1-6.

59. Lan Y. Effect of warm acupuncture on clinical pregnancy rate among IVF patients with Kidney-Yang Deficiency. World Latest Med Inform Digest . (2019) 19:191-3.

60. Liu YM,Tang JH. A randomized controlled study on the success rate of warm acupuncture on tube baby in elderly patients with kidney-yang deficiency and its effect on menstruation and endometrial level. World Chin Med . (2019) 14:1880-3.

61. Ma JJ, Zhang QH. Clinical study of acupuncture to improve endometrial receptivity, anxiety and depression and pregnancy outcome in patients with repeated IVF-ET transplantation failure of kidney deficiency and liver stagnation. J Shanghai U Tradit Chin Med. (2019) 33:32-8.

62. Jin ZC, Zheng J, Xia M, Sun H, Liu J, Yang Q, et al. Study of acupuncture improving clinical pregnancy outcome and its mechanisms in IVF-ET patients. CJITWM . (2018) 38:1174-9.

63. Zhang LX, Lan RX, Zheng CY. Effect of pricking blood therapy on clinical outcomes of frozen-thawed embryo transfer cycles in patients with thin endometrium. J Reprod Med. (2018) 27:411-5.

64. Xu ZZ, Gao Y, Zhang FQ. Effects of acupuncture on ovarian blood supply and pregnancy outcomes 
in patients receiving assisted reproduction.J Acupunct Tuina Sci. (2018) 16:253-9.

65. Xu JL, Yang ZG, Qian J, Zhuang HN, Yang ZG, Huang K, et al. Effect of stage-based acupuncturemoxibustion on endometrial thickness after repeated implantation failure in IVF-ET. Shanghai J Acumo . (2018) 37:200-4.

66. Ma JJ, Zhang QH, Weng XC, Dai MC. Effect of warm acupuncture on endometrial receptivity and pregnancy outcome in IVF-ET patients with repeated transplantation failure of kidney-Yang deficiency.Shanghai J Acu-mo . (2018) 37:1339-44.

67. Gao XA, Chen MZ,Ma WM,Luo GQ,Pan JL. The clinical effect of acupuncture combined with traditional Chinese medicine (TCM) artificial cycle therapy on the treatment of in vitro fertilization (IVF)cycle therapy on the treatment of in vitro fertilization cycle on the treatment of poor ovarian response. J Foshan U . (2017) 35:57-61.

68. Wu R, Wang Y. Application time-point and effect observation of fire needling therapy in IVF-ET. Chin Acu-mo . (2017) 37:498-502.

69. Peng DH, Lin HC. Effect of acupuncture combined with chinese medicine therapy on endometrium and pregnancy outcome in patients with IVF-ET.Med Innov China. (2017) 14:121-25.

70. Wang XM, Tian LJ, Yuan HL, Liu H, Fang LN, Zhou Y. Curative observation of warming acupuncture treatment for the impact on receptivity of endometrium of embryo transfer patients. J Sichuan Traditional Chin Med . (2017) 35:184-7.

71. Luo GQ, Su WW, Ma WM, Situ YQ, Xie CJ, Yang MQ. The effect of warm needling pretreatment for endometrial receptivity of frozen embryo transfer. Chin Acu-mo. (2017) 37:831-5.

72. Chen Q, Hau C. Impacts on pregnancy outcome treated with acupuncture and moxibustion in IVF-ET patients. Chin Acup Moxib. (2015) 35:313-7.

73. Wang X, Jing F, Wang C, Tian L, Liu H, Fang L, et al. The influence for success rate of warm acupuncture for tube baby of infertility patients with kidney yang deficiency: a randomized controlled trial.Chin Acu-mo. (2016) 36:906-10.

74. Yan HL,He SZ,Xing YJ, Luo XD, Lin B,Lai HH. Clinical study of embryo transfer techniquesacupuncture treatment in vitro fertilization. Guangzhou Med . (2015) 46:13-6.

75. Gu SY, Tian Q, Hu YJ, Zhang ZL. Clinical observation of acupuncture for Nourishing Shen-Gan and Chong-Ren channels on patients being in vitro fertilization-embryo transfer. Tianjin J Tradit Chin Med . (2012) 29:440-2.

76. Cui W, Li J, Sun W, Wen J. Effect of electroacupuncture on oocyte quality and pregnancy for patients with PCOS undergoing in vitro fertilization and embryo transfervitro fertilization and embryo transfer. Chin Acu-mo . (2011) 31:687-91.

77. Zhang M, Huang G, Lu F, Paulus WE, Sterzik K. Effects of acupuncture on the pregnancy rate in embryo transfer and mechanisms. Chin Acu-mo 2003; 23:7-9.

78. Chen H, Yang M, Lao L. Acupuncture for women undergoing in vitro fertilization. JAMA. (2018) 320:1385.

79. Zheng C, Wan X, Xu X, Zhang M, Huang G, Wang W. General issues in clinical research of acupuncture and in vitro fertilization.Evid Based Complement Alternat Med. (2020) 2020:3460641.

\section{Table/Figure Caption List}

Table 1. The overall and stratified results of CPR and LBR outcomes by acupuncture type

$\mathrm{CI}=$ confidence interval. $\mathrm{CPR}=$ clinical pregnancy rate. $\mathrm{EA}=$ electric acupuncture. $\mathrm{LA}=$ laser acupuncture. $\mathrm{LBR}=$ live birth rate. $\mathrm{OR}=$ odds ratio. $\mathrm{TA}=$ traditional acupuncture. TEAS $=$ Transcutaneous electrical acupoint stimulation. $\mathrm{WA}=$ warm acupuncture.

Table 2. The results of subgroup analyses for CPR outcomes of TA and TEAS treatment

$\mathrm{CI}=$ confidence interval. $\mathrm{OR}=$ odds ratio. $\mathrm{RIF}=$ repeated $\mathrm{IVF}$ failure. $\mathrm{TA}=$ traditional acupuncture. $\mathrm{TEAS}=$ Transcutaneous electrical acupoint stimulation. 


\section{Table 3. The results of analyses for LBR outcomes of TA and TEAS treatment}

$\mathrm{CI}=$ confidence interval. $\mathrm{OR}=$ odds ratio. $\mathrm{ET}=$ embryo transfer. $\mathrm{RIF}=$ repeated $\mathrm{IVF}$ failure. $\mathrm{TA}=$ traditional acupuncture. TEAS $=$ Transcutaneous electrical acupoint stimulation.

\section{Hosted file}

Table.docx available at https://authorea.com/users/437790/articles/539183-pregnancy-benefitof-acupuncture-on-in-vitro-fertilization-a-systematic-review-and-meta-analysis 